\title{
INSTRUMENTOS QUE AVALIAM A ESPIRITUALIDADE DE PACIENTES EM CUIDADOS PALIATIVOS: REVISÃO INTEGRATIVA DA LITERATURA
}

\author{
INSTRUMENTS EVALUATING THE SPIRITUALITY OF PATIENTS IN PALLIATIVE CARE: \\ INTEGRATING REVIEW OF THE LITERATURE
}

\section{RESUMO}

A dimensão espiritual é um aspecto de grande relevância na assistência paliativa, embora seja de difícil abordagem por parte dos profissionais. Desse modo, o estudo tem o objetivo de investigar os instrumentos que avaliam a espiritualidade de pacientes sob cuidados paliativos na literatura científica. Trata-se de uma revisão integrativa da literatura realizada mediante busca na Biblioteca Virtual Scientific Eletronic Library Online, nas bases de dados Literatura Latino-Americana e do Caribe em Ciências da Saúde e Índice Bibliográfico Español en Ciencias de la Salud, utilizando os descritores cuidados paliativos and espiritualidade. Fizeram parte da amostra 7 estudos. A maior parte foi publicada na revista Psicooncología $(28,57 \%)$, era de revisão (57,14\%), e foi publicado em 2015 e 2016 (28,57\%). As pesquisas apontaram vinte e oito instrumentos diferentes que avaliam a espiritualidade de pacientes em cuidados paliativos. Os instrumentos que avaliam a espiritualidade são ferramentas úteis para auxiliar os pacientes que apresentam doenças ameaçadoras da vida, entretanto apenas alguns instrumentos são direcionados especificamente para pacientes em cuidados paliativos.

Palavras-chave: Cuidados paliativos. Pacientes. Espiritualidade.

\section{ABSTRACT}

The spiritual dimension is an aspect of great relevance in palliative care, although it is difficult for professionals to approach. Thus, the study aims to investigate the instruments that evaluate the spirituality of patients undergoing palliative care in the scientific literature. This is an integrative review of the literature carried out by

\footnotetext{
1 Graduado em Enfermagem pelo Centro Universitário de João Pessoa (UNIPÊ). E-mail: jorgeneto87@hotmail.com

2 Mestre em Enfermagem pela Universidade Federal da Paraíba (UFPB). Docente do Centro Universitário de João Pessoa (UNIPÊ). E-mail: carla.evangelista@unipe.edu.br

3 Mestre em Enfermagem pela Universidade Federal da Paraíba (UFPB). Docente do Centro Universitário de João Pessoa (UNIPÊ). E-mail: ronny.cruz@unipe.edu.br

4 Especialista em Enfermagem do Trabalho pela Faculdade Integrada de Patos. E-mail: biana_biana@hotmail.com
} 
searching the Scientific Eletronic Library Online Virtual Library, the Latin American and Caribbean Literature in Health Sciences databases and the Spanish Bibliographical Index in Health Sciences, using the descriptors palliative care and spirituality. Seven studies were included in the sample. The majority was published in the journal Psicooncología (28.57\%), review era (57.14\%), and was published in 2015 and 2016 $(28.57 \%)$. The surveys pointed out twenty-eight different instruments that evaluate the spirituality of patients in palliative care. The instruments that evaluate spirituality are a useful tool to help patients with life threatening diseases, however only a few instruments are specifically aimed at patients in palliative care.

Keywords: Palliative care. Patients. Spirituality.

\section{INTRODUÇÃO}

Nos últimos anos os cuidados paliativos entraram em evidência e vem sendo discutido por pesquisadores nacionais e internacionais. Isso se deve ao fato do grande número de doenças diagnosticadas como crônicas e incuráveis e da necessidade de cuidados que proporcionem uma melhor qualidade de vida mesmo diante da impossibilidade de cura.

Os cuidados paliativos são definidos como cuidados direcionados a clientes que apresentam doenças ameaçadoras da vida, e proporcionam a melhoria da qualidade de vida, a partir de cuidados para a prevenção e redução da dor, sofrimento e sintomas físicos, sociais, psicológicos e espirituais (INCA, 2017).

No que diz respeito à espiritualidade, esta é definida como uma busca de significado e propósito para a vida, em dimensões que transpõem o mundo material, levando o ser humano a experiência de algo maior que o existencial, podendo ou não estar relacionada a uma prática religiosa (TAVARES, 2013).

Estudo verificou que a espiritualidade é uma ferramenta considerada importante na assistência paliativa por promover a melhora do quadro clínico do paciente e auxiliar na aceitação e no enfrentamento deles diante do adoecimento. Os enfermeiros participantes da pesquisa compreenderam a espiritualidade como fonte de força, conforto e fé (EVANGELISTA et al., 2016).

No entanto, muitos profissionais não conseguem atendera dimensão espiritual dos que se encontram sob seus cuidados, trazendo à tona a necessidade de uma melhor assistência espiritual durante o processo de morte e do desenvolvimento de instrumentos que avaliem os aspectos espirituais de pacientes que se encontram em cuidados paliativos (GARCÍA, 2016; GALIANA et al., 2016).

Desse modo, o estudo tem o objetivo de investigar os instrumentos que avaliam a espiritualidade de pacientes em cuidados paliativos na literatura científica. 


\section{REVISÃO BIBLIOGRÁFICA}

O cuidar de pessoas em processo de finitude é uma prática antiga e que vinha sendo realizada muito antes da existência do cuidado paliativo. Ressalta-se que o termo "paliativo" deriva do "pallium" que diz respeito a um manto que era utilizado pelos peregrinos como forma de proteção contra tempestades durante viagens direcionadas aos santuários. Por isso, este cuidado destina-se a proteger o indivíduo do sofrimento que pode ser evitado (MACIEL et al., 2016).

A história dos cuidados paliativos remete a tradição religiosa, de acompanhamento de moribundos, e de atos concretos para aliviar o sofrimento diante das doenças incuráveis (MARQUES, 2014). Logo, estes cuidados surgiram para preencher a lacuna na assistência direcionada a pacientes com doenças potencialmente fatais (MATSUMOTO, 2012). Além disso, influenciaram a realização de novas condutas por parte dos profissionais direcionadas a morte, morrer e ao luto (MACIEL et al., 2016).

No mundo contemporâneo, foi desenvolvido inicialmente por Cicely Saunders, na década de 50, enquanto assistia pacientes em finitude, e elaborou conhecimentos e práticas para auxiliar nos cuidados destinados a este público. Destaca-se que Saunders possuía três formações acadêmicas: medicina, enfermagem e serviço social (ACADEMIA NACIONAL DE CUIDADOS PALIATIVOS, 2018).

A primeira definição dos cuidados paliativos, que consistia em cuidados ativos e totais destinados a pacientes cuja doença não respondia aos tratamentos curativos, foi publicada pela Organização Mundial de Saúde em 1990, a partir de um relatório elaborado por um comitê de especialistas em atenuação da dor, oncologia e cuidados paliativos (OMS, 1990; GÓMEZ-BATISTE; CONNOR, 2017).

Entretanto, em 2002 a definição foi revisada no intuito de ampliar o conceito para todas as doenças ameaçadoras da vida, não se restringindo apenas ao câncer, e exigindo, sempre que possível, que a assistência pudesse ser fornecida junto ao tratamento curativo (GÓMEZ-BATISTE; CONNOR, 2017).

Neste contexto, a atenção paliativa se direciona a melhoria da qualidade de vida de pacientes que possuem doenças ameaçadoras da vida e seus familiares, mediante a prevenção e redução da dor e dos sintomas físicos, psicológicos, sociais e espirituais. Deve ser aplicado no início da doença, juntamente com terapias que visam o prolongamento da vida (CONNOR; BERMEDO, 2014), a pacientes com doenças crônicas e ameaçadoras da vida, nos diversos ambientes e níveis de atenção à saúde, sejam eles primários, secundários ou terciários (GÓMEZ-BATISTE ef al., 2017).

Em 2018, houve um avanço nos cuidados paliativos no Brasil, com a publicação da Resolução $n^{\circ} 41$, de 31 de outubro, que trata das diretrizes para organização dos serviços de assistência paliativa. De acordo com a Resolução, esta 
assistência envolve uma equipe multidisciplinar, e tem como objetivo primordial a melhoria da qualidade de vida de pacientes e familiares perante doença ameaçadora da vida, seja aguda ou crônica, a partir do seu diagnóstico (BRASIL, 2018).

Assim, a equipe de cuidados paliativos deve ser composta por profissionais das variadas áreas do conhecimento, a exemplo da medicina, enfermagem, serviço social, psicologia, terapia ocupacional, fonoaudiologia, fisioterapia e nutrição. Além de capelães e voluntários com habilidades para o cuidado de pacientes com doenças que ameaçam a vida (SOCIEDADE BRASILEIRA DE GERIATRIA E GERONTOLOGIA, 2015).

No Brasil, os cuidados paliativos podem ser encontrados em várias regiões, com destaque para o sudeste que possui uma média de 177 instituições que realizam cuidados paliativos e que são cadastradas na Academia Nacional de Cuidados Paliativos, seguidos do sul com 60, nordeste com 52, centro-oeste com 25, e norte com 9 (ACADEMIA NACIONAL DE CUIDADOS PALIATIVOS, 2019). E apesar do crescimento dos programas direcionados a esses cuidados no ambiente hospitalar, nem todos hospitais possuem unidades e equipes especializadas no tratamento paliativo. Além disso, muitos profissionais não conhecem e não realizam a assistência paliativa (MACIEL et al., 2016).

Os cuidados paliativos reafirmam vida e entendem a morte como algo natural; integram as dimensões psicológicas, sociais e espirituais na assistência; não apressam ou adiam a morte; oferecem auxilio para o paciente e sua família diante do problema de saúde; utilizam uma abordagem interdisciplinar na prestação de cuidados incluindo aconselhamento e suporte ao luto, com o objetivo de melhorar a qualidade de vida naqueles que se encontram com uma doença potencialmente fatal (INCA 2017; MATSUMOTO, 2012; HERMES; LAMARCA, 2013).

Diante de uma doença que ameaça a vida, os pacientes e familiares passam por situações difíceis e que não estavam preparados, o que leva a angústia, depressão e falta de esperança, sendo importante a abordagem desses aspectos sob o ponto de vista da espiritualidade (MATSUMOTO, 2012).

A palavra "espírito" tem origem no hebraico e grego, cujo significado representa o ar em movimento, hálito ou vento. Desse modo, é considerada o princípio da vida, energia vital, sede de sentimento, pensamentos e decisões, de maneira a dar força aos indivíduos diante de algumas situações (TAVARES et al., 2016).

A espiritualidade é universal, inerente a todo indivíduo, e dá sentido a existência humana. É uma presença diária, e está presente no meio social, relacional, profissional, na educação, saúde, lazer, religião, no íntimo de cada ser, entre ateus, agnósticos e religiosos, ou seja, em todos os espaços e realidades existenciais (TAVARES et al., 2016). 
A assistência prestada pelos profissionais de saúde deve contemplar esta dimensão auxiliando na promoção do conforto e no respeito as necessidades dos clientes e cuidadores, para tanto, estes deverão realizar uma abordagem adequada, diagnosticando a espiritualidade, intervindo e avaliando-a (VIANA; SOUZA, 2017).

A necessidade de avaliar a atenção espiritual e de fornecer recursos para seu desenvolvimento levou a criação de vários instrumentos específicos. Atualmente, cresce a publicação de instrumentos para avaliação e acompanhamento espiritual, sendo aplicados em diferentes populações e com a utilização de várias abordagens (GALIANA et al., 2016).

Desse modo, faz-se necessário o conhecimento destes instrumentos, de modo a permitir uma melhor abordagem espiritual no atendimento dos pacientes que se encontram em cuidados paliativos.

\section{METODOLOGIA}

Trata-se de uma revisão integrativa da literatura, que foi operacionalizada a partir das seis etapas sugeridas por Mendes, Silveira e Galvão (2008): identificação do tema e seleção de hipótese/questão norteadora; estabelecimento de critérios de inclusão e exclusão/amostragem ou busca na literatura; definição das informações a serem extraídas dos estudos, avaliação dos estudos incluídos; interpretação dos resultados; e apresentação da revisão.

\section{PRIMEIRA ETAPA: IDENTIFICAÇÃO DO TEMA E DEFINIÇÃO DA QUESTÃO NORTEADORA}

Para investigar os instrumentos que abordam a espiritualidade de pacientes que se encontram em cuidados paliativos formulou-se a seguinte questão norteadora: Quais os instrumentos são utilizados para avaliar a espiritualidade de pacientes em cuidados paliativos na literatura científica?

\section{SEGUNDA ETAPA: ESTABELECIMENTO DE CRITÉRIOS DE INCLUSÃO E EXCLUSÃO/AMOSTRAGEM E BUSCA NA LITERATURA}

Para a seleção dos estudos desta revisão integrativa, os critérios de inclusão estabelecidos foram: artigos disponíveis na integra, no idioma português e espanhol, que estivessem presentes na biblioteca Scientific Electronic Library Online (Scielo), Índice Bibliográfico Español en Ciencias de la Salud (IBECS) e na Literatura Latino-americana e do Caribe em Ciências da Saúde (LILACS), que abordassem a temática proposta, dentro do período de 2005 a 2017. 
Os critérios de exclusão envolveram artigos com ano de publicação inferior a 2005, capítulos de livros, monografia, dissertações, teses, textos não científicos e artigos científicos sem disponibilidade do texto na íntegra.

A busca foi realizada nos meses de setembro e outubro 2017 através das bibliotecas e bases mencionadas. Inicialmente utilizou-se a terminologia em saúde consultada nos Descritores em Ciências da Saúde (DeCS): cuidados paliativos, espiritualidade e escalas. Entretanto, devido ao pequeno quantitativo de artigos encontrados optou-se por trabalhar com o cruzamentos dos descritores cuidados paliativos e espiritualidade para seleção dos artigos, com o auxilio do operador booleano "AND" para realizar a estratégia de busca, de forma a abranger um maior número de publicações.

\section{TERCEIRA ETAPA: DEFINIÇÃO DAS INFORMAÇÕES A SEREM EXTRAIIDAS DOS ESTUDOS}

Para definir os elementos extraídos do estudo foi elaborado um instrumento de coleta de dados constando dos seguintes itens: ano de publicação, título do artigo, periódico, delineamento de pesquisa, país de desenvolvimento do estudo, título do instrumento e descrição do instrumento.

\section{QUARTA ETAPA: AVALIAÇÃO DOS ESTUDOS INCLUÍDOS}

Nesta etapa, foi feita a avaliação dos estudos mediante leitura do texto na íntegra e análise crítica do conteúdo.

\section{QUINTA ETAPA: INTERPRETAÇÃO DOS RESULTADOS}

Os resultados da presente revisão integrativa da literatura foram interpretados a luz da literatura.

\section{SEXTA ETAPA: APRESENTAÇÃO DA REVISÃO INTEGRATIVA}

A apresentação envolveu a descrição da revisão integrativa propriamente dita, a presença de quadros e a síntese do conhecimento sobre a temática investigada.

\section{RESULTADOS E DISCUSSÃO}

Esta revisão integrativa foi constituída por um total de sete publicações que versaram sobre a temática "Instrumentos que avaliam a espiritualidade de pacientes em cuidados paliativos", conforme caracterização explicitada no Quadro 1. 
Quadro 1 - Distribuição dos estudos incluídos na revisão integrativa de acordo com título, periódico, ano, delineamento e país.

\begin{tabular}{|c|c|c|c|c|}
\hline $\mathbf{N}$ & TITULO & PERIÓDICO/ANO & DELINEAMENTO & LOCAL \\
\hline 1 & $\begin{array}{l}\text { A importância da integração } \\
\text { da espiritualidade e da } \\
\text { religiosidade no manejo da } \\
\text { dor e dos cuidados paliativos }\end{array}$ & $\begin{array}{c}\text { Revista de psiquiatria } \\
\text { clínica/2007 }\end{array}$ & Revisão & Brasil \\
\hline 2 & $\begin{array}{l}\text { Bienestar espiritual de } \\
\text { enfermos erminales y de } \\
\text { personas aparentemente } \\
\text { sanas }\end{array}$ & $\begin{array}{l}\text { Investigación Y } \\
\text { Educación En } \\
\text { Enfermería/2009 }\end{array}$ & $\begin{array}{l}\text { Quantitativo, } \\
\text { descritivo, } \\
\text { comparativo. }\end{array}$ & Colômbia \\
\hline 3 & $\begin{array}{l}\text { Cuidados paliativos e } \\
\text { espiritualidade: revisão } \\
\text { integrativa da literatura }\end{array}$ & $\begin{array}{l}\text { Revista Brasileira de } \\
\text { Enfermagem/2016 }\end{array}$ & Revisão & Brasil \\
\hline 4 & $\begin{array}{l}\text { Sentido vital en la enfermedad } \\
\text { avanzada: desarrollo de } \\
\text { una herramienta para guiar } \\
\text { la atención psicosocial y } \\
\text { espiritual en el paciente y } \\
\text { família }\end{array}$ & Psicooncología/2015 & Metodológico & Espanha \\
\hline 5 & $\begin{array}{l}\text { Enfermería y necesidades } \\
\text { espirituales en el paciente } \\
\text { con enfermedad en etapa } \\
\text { terminal }\end{array}$ & $\begin{array}{l}\text { Enfermería: Cuidados } \\
\text { Humanizados/2016 }\end{array}$ & Reflexivo & Espanha \\
\hline 6 & $\begin{array}{l}\text { Cuestionarios de atención } \\
\text { espiritual en cuidados } \\
\text { paliativos: revisión de la } \\
\text { evidencia para su aplicación } \\
\text { clínica }\end{array}$ & Psicooncología/2016 & Revisão & Espanha \\
\hline 7 & $\begin{array}{l}\text { Significados da espiritualidade } \\
\text { para pacientes com câncer } \\
\text { em cuidados paliativos }\end{array}$ & $\begin{array}{c}\text { Estudos de } \\
\text { Psicologia/2017 }\end{array}$ & Revisão & Brasil \\
\hline
\end{tabular}

Fonte: Dados da pesquisa, 2017.

A produção cientifica acerca da temática esteve presente em 6 periódicos diferentes. A revista Psicooncología obteve 02 (28,57\%) artigos sobre a temática, enquanto as demais revistas tiveram apenas 01 (14,29\%) publicação e são elas: Enfermería: Cuidados Humanizados, Estudos de Psicologia, Investigación y Educación en Enfermería, Revista de psiquiatria clínica, Revista Brasileira de Enfermagem.

A psicooncología, revista que obteve destaque de publicações, é uma revista interdisciplinar da Universidad Complutense, Sociedad Española Oncología Médica (SEOM) e Sociedad Española de Psicooncología (SEPO), que possui publicação trimestral, e foi criada em 2003, na Espanha. A revista tem objetivo de publicar estudos teóricos, experimentais e clínicos referentes aos aspectos psicológicos, sociais, éticos e a qualidade de vida associada ao câncer (RCC, 2017). O fato da revista ter como foco o câncer, uma das doenças beneficiadas pela assistência paliativa justifica o número de publicações sobre a temática.

Quanto ao ano de publicação, destaca-se que o ano de 2015 e 2016 foram considerados os anos de maiores produções de artigos sobre a temática, com 2 
(28,57\%) publicações, cada; seguido dos anos de 2007, 2009 e 2017 com apenas 01 (14,29\%) publicação. Nos demais anos constatou-se um período de latência com ausência de publicações. Desse modo, foi possível perceber que a produção ainda é tímida considerando os critérios elencados para a busca.

Entretanto, a partir de 2015 observou-se um pequeno crescimento das publicações, em decorrência das crescentes discussões sobre os cuidados paliativos e sobre a necessidade da prestação do cuidado integral principalmente no que diz respeito ao paciente com doença grave. Destaca-se que em 2017 evidenciou-se apenas uma publicação, embora o número possa aumentar em consequência de que o período de coleta de dados não contemplou o ano de 2017 por completo, uma vez que esse ainda estava em curso.

No que concerne ao delineamento de pesquisa, a maior parte dos estudos referem- se a estudos de revisão, com $4(57,14 \%)$ das produções, seguido das pesquisas quantitativa, metodológica e reflexiva com 1 (14,29\%), cada.

Estudo desenvolvido por Galiana et al. (2016) resumiu 15 artigos acerca de questionários de avaliação de cuidados espirituais em cuidados paliativos e a aplicabilidade clínica destes, apresentando um maior quântico de pesquisas de revisão.

A maioria dos estudos é internacional (57,14\%), com destaque para a Espanha. Cumpre assinalar que o Brasil publicou 3 artigos (42,85\%) sobre a temática no período de tempo investigado.

Os instrumentos que avaliam a espiritualidade de pacientes que se encontram em cuidados paliativos identificados nos estudos incluídos na presente revisão encontram-se no quadro 2.

Quadro 2 - Distribuição dos estudos incluídos na revisão integrativa de acordo os instrumentos que avaliam a espiritualidade.

\begin{tabular}{|l|l|}
\hline \multicolumn{1}{|c|}{ INSTRUMENTOS } & \multicolumn{1}{c|}{ DESCRIÇÃo } \\
\hline Brief-RCope & Avaliação do enfrentamento religioso e espiritual. \\
\hline $\begin{array}{l}\text { Cancer-specific geriatric } \\
\text { assessment tool }\end{array}$ & $\begin{array}{l}\text { Avaliação da população idosa com câncer, incluindo os } \\
\text { domínios socioeconômicos, sintomas físicos, comorbidade, estado } \\
\text { funcional, estado psicológico, apoio social, cognição, estado } \\
\text { nutricional e questões espirituais. }\end{array}$ \\
\hline FICA & Avaliação da história religiosa e espiritual. \\
\hline DUREL & $\begin{array}{l}\text { Mensuração de três das principais dimensões do envolvimento: } \\
\text { religiosidade organizacional; religiosidade não organizacional e } \\
\text { religiosidade intrínseca. }\end{array}$ \\
\hline Escala de Bienestar Espiritual & $\begin{array}{l}\text { Avaliação da dimensão existencial do bem-estar espiritual e a } \\
\text { dimensão religiosa }\end{array}$ \\
\hline $\begin{array}{l}\text { Escala de Perspectiva } \\
\text { Espiritual de Reed }\end{array}$ & $\begin{array}{l}\text { Mede o conhecimento de si mesmo, sensação de conexão } \\
\text { com um ser de natureza superior ou existência de um propósito } \\
\text { supremo }\end{array}$ \\
\hline
\end{tabular}

\section{INTER/SCIENTIA}

REVISTA INTERSCIENTIA | V. 7 | N. 2 | P. 160-173 | JUL-DEZ/2019 


\begin{tabular}{|c|c|}
\hline $\begin{array}{l}\text { Escala de valoración del } \\
\text { sufrimiento espiritual de } \\
\text { Ortega }\end{array}$ & $\begin{array}{l}\text { Avalia o bem-estar/sofrimento espiritual de pacientes com câncer } \\
\text { em fase terminal. }\end{array}$ \\
\hline FACIT-SP & Avalia o bem-estar espiritual de pacientes com doenças crônicas \\
\hline FACIT-Sp-12 & Avalia o bem-estar espiritual de pacientes com doenças crônicas \\
\hline $\begin{array}{l}\text { FCG spiritual well-being was } \\
\text { measured using the COH- } \\
\text { QOL-FCG spiritual well-being } \\
\text { subscale }\end{array}$ & $\begin{array}{l}\text { Avalia o bem-estar espiritual de pacientes oncológicos em } \\
\text { cuidados paliativos. }\end{array}$ \\
\hline $\begin{array}{l}\text { Functional Assessment of } \\
\text { Cancer Therapy-General } \\
\text { (FACT-G) quality-of-life scale } \\
\text { with a 12-item spiritual well- } \\
\text { being subscale. }\end{array}$ & $\begin{array}{l}\text { Avalia os impactos da terapia do câncer em quatro domínios: } \\
\text { físico, social/familiar, emocional e funcional. }\end{array}$ \\
\hline HOPE & Mede a esperança dos doentes em cuidados paliativos. \\
\hline $\begin{array}{l}\text { JAREL Spiritual Well-Being } \\
\text { Scale }\end{array}$ & $\begin{array}{l}\text { Ferramenta de avaliação para que fornece o diagnóstico de } \\
\text { enfermagem referente a espiritualidade para ser utilizada em } \\
\text { adultos mais velhos. }\end{array}$ \\
\hline $\begin{array}{l}\text { Measure of anger toward } \\
\text { God }\end{array}$ & $\begin{array}{l}\text { Avalação da negação de pacientes terminais em relação a } \\
\text { Deus. }\end{array}$ \\
\hline $\begin{array}{l}\text { Missoula- VITAS Quality ofLife } \\
\text { Index }\end{array}$ & $\begin{array}{l}\text { Descreve a experiência qualitativa e subjetiva de qualidade } \\
\text { vida em pacientes sob cuidados paliativos. A escala possui as } \\
\text { seguintes dimensões: sintomas, função, interpessoal, bem-estar e } \\
\text { transcendência. }\end{array}$ \\
\hline $\begin{array}{l}\text { Palliative Care Outcome } \\
\text { Scale (POS) }\end{array}$ & $\begin{array}{l}\text { É uma escala multidimensional de avaliação de Qualidade de } \\
\text { Vida de pessoas em Cuidados Paliativos. }\end{array}$ \\
\hline $\begin{array}{l}\text { Palliative Care Problem } \\
\text { Severity Score }\end{array}$ & $\begin{array}{l}\text { Avalia a gravidade do problema em quatro domínios de } \\
\text { cuidados paliativos (dor, outros sintomas, problemas psicológicos/ } \\
\text { espirituais, familiares). }\end{array}$ \\
\hline $\begin{array}{l}\text { Schedule for Meaning in Life } \\
\text { (SMILE) }\end{array}$ & $\begin{array}{l}\text { Avalia o significado da vida e a satisfação em várias áreas } \\
\text { incluindo a espiritual }\end{array}$ \\
\hline $\begin{array}{l}\text { Self-Transcendence Scale } \\
\text { (STS) }\end{array}$ & $\begin{array}{l}\text { Identifica questões intrapessoais, } \\
\text { experiências interpessoais, transpessoais e temporais }\end{array}$ \\
\hline SPIRIT & $\begin{array}{l}\text { Guia para identificar componentes importantes da história } \\
\text { espiritual. }\end{array}$ \\
\hline Spiritual Comfort Measure & Avalia a medição do conforto espiritual. \\
\hline Spiritual Distress Scale (SDS) & $\begin{array}{l}\text { Avalia angústia espiritual de pacientes com câncer, dividida em } \\
\text { quatro sub-escalas. }\end{array}$ \\
\hline $\begin{array}{l}\text { Spiritual Health Inventory } \\
\text { (SHI) }\end{array}$ & $\begin{array}{l}\text { Avalia se os pacientes estão satisfazendo suas necessidades } \\
\text { espirituais de auto-realização, relacionamentos e esperança. }\end{array}$ \\
\hline $\begin{array}{l}\text { Spiritual Involvement and } \\
\text { Beliefs Scale }\end{array}$ & $\begin{array}{l}\text { Fornece um método quantitativo de investigação sobre a } \\
\text { espiritualidade de um paciente, de forma a ser mais inclusivo de } \\
\text { outras religiões. }\end{array}$ \\
\hline $\begin{array}{l}\text { Spiritual Needs Inventory } \\
\text { (SNI) }\end{array}$ & $\begin{array}{l}\text { Instrumento para medir as necessidades espirituais dos pacientes } \\
\text { perto do fim da vida. }\end{array}$ \\
\hline $\begin{array}{l}\text { Spiritual Perspective Scale } \\
\text { (SPS) }\end{array}$ & Avalia a percepção sobre questões espirituais \\
\hline $\begin{array}{l}\text { Spiritual Well-Being Scale } \\
\text { (SWBS) }\end{array}$ & Avalia o bem estar espiritual de pessoas religiosas e não religiosas. \\
\hline
\end{tabular}

INTER/SCIENTIA

REVISTA INTERSCIENTIA | V. 7 | N. 2 | P. 160-173 | JUL-DEZ/2019 
Fonte: Dados da pesquisa, 2017.

Foram encontrados 28 instrumentos que avaliam a espiritualidade de pacientes em cuidados paliativos. De uma forma geral, as medidas relacionadas à espiritualidade e religiosidade perante uma doença acabam sendo algo indispensável para o paciente (HERRERRA, 2009; GARCIA, 2016).

Nesse contexto, é que autores buscam criar instrumentos que avaliam a espiritualidade, e cada um desses possuem características diferentes, como por exemplo, a FACIT-Sp e o FACIT-SP 12. OFACIT-Sp 12é um dos instrumentos mais utilizados e validados a nível mundial para avaliar o bem-estar espiritual de pacientes com doenças crônicas. Entretanto, enquanto o FACIT-SP possui 40 itens, abrangendo as dimensões física, social/familiar, emocional, funcional e preocupações adicionais, o FAIT-Sp 12 é uma versão reduzida que possui 12 itens (LUCCHETTI et al., 2015).

A Escala de Bem-estar Espiritual de Ellison possui 20 itens e é composta por subescalas relacionadas à dimensão existencial, religiosa, espiritual. Estudo realizado na Colômbia com 88 pessoas que foram divididas em dois grupos, um composto de pessoas com enfermidade crônica e morte eminente e o outro de pessoas saudáveis, mostrou que tanto as pessoas com enfermidade como as pessoas sãs tiveram um bem-estar espiritual, embora entre as saudáveis a média tenha sido maior. Os altos níveis dessa escala significam que as pessoas estão vivendo em harmonia interna (HERRERA, 2009).

Podem-se destacar duas das principais escalas que servem para avaliação da história espiritual de pacientes que se encontram em Cuidados paliativos, a escala FICA de autoria de Puchalski e a SPIRIT de Maugans. Ambas têm a função identificar os componentes importantes da história espiritual dos pacientes em cuidados paliativos. Essas escalas são simples e permitem uma avaliação rápida do paciente (ARAÙJO, 2011; SAPORETTI; SILVA, 2012).

Algumas escalas são direcionadas para pacientes com câncer, e são elas: Cancer-specific geriatric assessment tool, Escala de valoración del sufrimiento espiritual de Ortega, FCG spiritual well-being was measured using the COH-QOL-FCG spiritual well-being subscale, Spiritual Distress Scale (SDS), Spirituality Transcendence Measure (STM).

A Escala de valoración del sufrimiento espiritual de Ortega, é um instrumento de simples preenchimento que consta de dados pessoais, clínicos e referente ao sofrimento espiritual, que auxilia na abordagem e diagnóstico de sofrimento dos pacientes com câncer terminal, abrindo as portas para uma comunicação profunda com o paciente, e ajudando-os a encontrar sua paz interior mesmo diante da morte (ORTEGA GALÁN, 2008). 
Por sua vez, a escala de Medida de Transcendência de Espiritualidade (STM) avalia o bem estar espiritual de pacientes em estágio terminal e mensura três fontes de transcendência espiritual, o aspecto situacional, moral, biográfico e religioso (LEUNG; CHIU; CHEN, 2006).

Outras escalas como a FCG spiritual well-being was measured using the COH-QOL-FCG spiritual well-being subscale, HOPE, Measure of anger toward God, Missoula- VITAS Quality ofLife Index, Palliative Care Outcome Scale (POS), Palliative Care Problem Severity Score, Spiritual Needs Inventory (SNI), Spiritual Perspective Scale (SPS), são direcionadas especificamente para pacientes em cuidados paliativos.

A escala de Índice de Qualidade de Vida Missoula-Vitas (MVQOLI) é uma ferramenta exclusiva e especificamente projetada para medir a qualidade de vida de pacientes com doenças avançadas em um ambiente de cuidados paliativos (CORREIA; DE CARLO, 2012).

O Palliative Care Outcome Scale (POS) é uma escala de avaliação multidimensional da qualidade de vida bastante utilizada, no ensino, pesquisa e prática clínica, com pacientes que apresentam doenças crônico-degenerativa que se encontram em cuidados paliativos. O instrumento é uma importante medida de avaliação da qualidade de vida dos pacientes e familiares, da qualidade do atendimento prestado, e da organização de serviços atuantes em cuidados paliativos (RUGNO; CARLO, 2016; WENTLANDTET et al., 2012).

Utilizar instrumentos que avaliam a espiritualidade dos pacientes principalmente daqueles que se encontram com doença potencialmente fatal se torna indispensável, uma vez que auxilia o paciente no atendimento de suas necessidades espirituais que por vez são esquecidas pelos profissionais.

\section{CONSIDERAÇÕES FINAIS}

Percebe-se que os cuidados paliativos e a espiritualidade são dois componentes indispensáveis para os pacientes que estão sem possibilidades de recuperar a saúde através da terapêutica curativa, sendo a utilização de escalas que avaliam a dimensão espiritual uma ferramenta útil para verificar o bem-estar das pessoas que estão com uma enfermidade ameaçadora da vida e auxiliá-las durante a finitude.

O estudo evidenciou a existência de várias escalas que abordam a espiritualidade e que podem ser utilizadas com pacientes que se encontram com doença ameaçadora da vida, porém nem todos os instrumentos são específicos para pacientes em cuidados paliativos. Dentre os instrumentos que são específicos para esses pacientes, infere-se que alguns são utilizados diretamente com paciente que apresenta câncer, uma das doenças que pode ser favorecida pelo benefício da assistência paliativa 
É indispensável salientar que os aspectos relacionados à espiritualidade, fé e religiosidade são importantes para a melhoria da qualidade de vida em pacientes terminais e que devem ser atendidos pelos profissionais. Logo, urge a necessidade de que sejam desenvolvidas novas pesquisas relacionadas à temática, principalmente no âmbito nacional para que se tenha um maior conhecimento acerca do assunto. Também é válido destacar que seja instigado aos acadêmicos, o estudo dos instrumentos de avaliação da espiritualidade que estejam direcionados para os cuidados paliativos, para que possam ser preparados para atender a dimensão espiritual dos pacientes com doenças ameaçadoras da vida.

\section{REFERÊNCIAS}

ACADEMIA NACIONAL DE CUIDADOS PALIATIVOS (Brasil). Onde existem. São Paulo: Academia Nacional de Cuidados Paliativos, 2019. Disponível em: https://paliativo. org.br/ancp/onde-existem/ 2019. Acesso em: 07 dez. 2019.

ACADEMIA NACIONAL DE CUIDADOS PALIATIVOS (Brasil). Análise situacional e recomendações da ANCP para estruturação de programas de cuidados paliativos no Brasil. São Paulo: Academia Nacional de Cuidados Paliativos, 2018.

ARAÚJO, M.Â.M. O cuidado espiritual: O modelo à luz da análise existencial e da relação de ajuda. 2011.195 f. Tese (Doutorado em Enfermagem) - Programa de PósGraduação em Enfermagem, Universidade Federal do Ceará, Fortaleza, 2011.

BRASIL. Ministério da Saúde. Resolução n 41, de 31 de outubro de 2018. Dispõe sobre as diretrizes para a organização dos cuidados paliativos, à luz dos cuidados continuados integrados, no âmbito Sistema Único de Saúde (SUS). Brasília: Ministério da Saúde, 2018. Disponível em: https://portalarquivos2.saude.gov.br/images/ pdf/2018/novembro/23/RESOLUCAO-N41.pdf

CONNOR, S.R.; BERMEDO, MCS. Global atlas of palliative care at the end of life. Genebra: World Health Organization, 2014.

CORREIA, F.R.; DE CARLO, M.M.R.P. Avaliação de qualidade de vida no contexto dos cuidados paliativos: revisão integrativa de literatura. Rev. Latino-Am. Enfermagem, $v$. 20, n. 2, p. 401-410, 2012. Disponível em: http://www.scielo.br/scielo.php?script=sci_ arttext\&pid=S0104-11 692012000200025\&lng=en\&nrm=iso. Acesso em: 25 nov. 2017.

EVANGELISTA, C.B. et al. Cuidados paliativos e espiritualidade: revisão integrativa da literatura. Revista Brasileira de Enfermagem, v. 69, n. 3, p. 591-601, 2016. Disponível em: http://www.scielo.br/pdf/reben/v69n3/0034-7167-reben-69-03-0591.pdf. Acesso em: 10 nov. 2017. 
GALIANA, L. et al. Cuestionarios de atención espiritual en cuidados paliativos: revisión de la evidencia para su aplicación clínica. PSICOONCOLOGÍA, v. 13, n.2-3, p. 385-397, 2016.

GARCIA, E.P. Enfermería y necessidades espiritualies em el paciente con enfermidade em etapa terminal. Enfermería, v. 5, n. 2, p. 41-45, 2016. Disponível em: http://www. scielo.edu.uy/scielo.php?script=sci_arttext\&pid=\$2393-66062016000200006\&lng=es\& nrm=iso. Acesso em 10 nov. 2017.

GÓMEZ-BATISTE, X; CONNOR, S (org.). Building Integrated Palliative Care Programs and Services. Geneva: WHO Collaborating Centre Public Health Palliative Care Programmes, 2017.

HERMES, Hélida Ribeiro; LAMARCA, Isabel Cristina Arruda. Cuidados paliativos: uma abordagem a partir das categorias profissionais de saúde. Ciência e Saúde Coletiva, v. 18, n. 9, p. 2577-2588, 2013. Disponível em: http://www.scielo.br/scielo. php?script=sci_arttext\&pid=S1413-81232013000900012. Acesso em 11 set. 2017.

HERRERA, B.S. Bienestar espiritual de enfermos terminalesy de personas aparentemente sanas. Invest. educ. enfermagem, v. 27, n. 1, p. 86-95, 2009. Disponível em: http:// www.scielo.org.co/scielo.php?script=sci_arttext\&pid=S0120-53072009000100009\&lng =es\&nrm=iso. Acesso em 10 nov. 2017.

INSTITUTO NACIONAL DO CÂNCER. Cuidados paliativos. Rio de janeiro, 2017. Disponível em:http://www2.inca.gov.br/wps/wcm/connect/cancer/site/tratamento/ cuidados_paliativos. Acesso em: 06 nov 2017.

LEUNG, K.K.; CHIU, T.Y.; CHEN, C.Y. The Influence of Awareness of Terminal Condition on Spiritual Well-Being in Terminal Cancer Patients. JPain Symptom Manage, v. 31, n. 5, p. 449-456, 2006.

LUCCHETTI G. et al. Validation of the Portuguese Version of the Functional Assessment of Chronic Illness Therapy-Spiritual Well-Being Scale (FACIT-Sp 12) Among Brazilian Psychiatric Inpatients. J Relig Health. v. 54, n. 1, p. 112-21, 2015. Disponível em: https:// doi.org/10.1007/s10943-013-9785-z. Acesso em: 10 ago 2017.

MACIEL, AP da S. et al. Referências históricas: definições e princípios em cuidados paliativos. In: VICENSI, M DO C (org.). Enfermagem em cuidados paliativos. Florianópolis: Conselho Regional de Enfermagem de Santa Catarina: Letra Editorial, 2016.

MARQUES, A.L. História dos cuidados paliativos em Portugal: raízes. Cuidados Paliativos, v. 1, n.1, p. 7-12, 2014.

MATSUMOTO, D. Y. Cuidados Paliativos: conceito, fundamentos e princípios. In: Academia Nacional de Cuidados Paliativos. Manual de Cuidados paliativos. 3. ed. 
Diagraphic. Rio de Janeiro. 2012.

MENDES, K.D.S.; SILVEIRA, R.C.C.P.; GALVÃO, C.M. Revisão integrativa: método de pesquisa para a incorporação de evidências na saúde e na enfermagem. Texto contexto - enferm. vol. 17, n. 4, p. 758-64, 2008. Disponível em: http://www.scielo. br/scielo.php?pid=S010407072008000400018\&script=sci_arttext. Acesso em: 01 maio 2017.

ORTEGA GALÁN, A.M. Validación de una escala valorativa del diagnóstico enfermero "Sufrimiento espiritual" en los enfermos oncológicos en fase terminal. Biblioteca Lascasas, v. 4, n. 1, p. 1-16, 2008. Disponível em: http://www.index-f.com/lascasas/ documentos/lc0309.php. Acesso em: 03 nov 2017.

RCC. Revistas Científicas Complutenses. Psioconcologia. Madrid: 2003. ISSN 16967240 .

RUGNO, F.C.; CARLO, M.M.R.P. The Palliative Outcome Scale (POS) applied to clinical practice and research: an integrative review. Rev. Latino-Am. Enfermagem, v. 24, e2764, 2016. Disponível em: http://dx.doi.org/10.1590/1518-8345.0993.2764. Acesso em: 01 dez. 2017.

SAPORETTI, L.A.; SILVA, A.M.O.P. Aspectos particulares e ritos de passagem nas diferentes religiões. In: Academia Nacional de Cuidados Paliativos. Manual de Cuidados paliativos. 3. ed. Diagraphic. Rio de Janeiro. 2012.

SOCIEDADE BRASILEIRA DE GERIATRIA E GERONTOLOGIA. Vamos falar de Cuidados Paliativos. Rio de Janeiro: Sociedade Brasileira de Geriatria e Gerontologia, 2015.

TAVARES, CÁSSIA QUELHO et al. Espiritualidade, religiosidade e saúde: velhos debates, novas perspectivas. INTERAÇÕES, v.11, n. 20, p. 85-97, 2016.

TAVARES, C.Q. Espiritualidade e bioética: prevenção da "violência" em instituições de saúde. Rev Pistis Prax, Teol Pastor, v. 5, n. 1, p. 39-57, 2013.

OMS. Organización Mundial de la Salud. Alívio del dolor e tratamiento paliativo en el cancer: informe de un comité de expertos de la OMS. Genebra: OMS, 1990.

VIANNA, MARIA LEONOR GOMES de SÁ; SOUZA, WALDIR. A Espiritualidade dos cuidadores informais de pacientes em cuidados paliativos: uma reflexão bioética na perspectiva da alteridade. Estudos Teológicos, v. 57 n. 2 p. 401-413, 2017.

WENTLANDT, K. et al. Referral practices of oncologists to specialized palliative care. J Clin Oncol, v. 30, n. 35, p. 4380-4386, 2012. 